\title{
Cerebrovascular Risk in Restless Legs Syndrome: Intima-Media Thickness and Cerebral Vasomotor Reactivity: A Case-Control Study
}

\author{
Francesco Janes $\mathbb{D}^{\prime}$ \\ Simone Lorenzut ${ }^{\prime}$ \\ Francesca Bevilacqua ${ }^{2}$ \\ Stefano de Biase ${ }^{2}$ \\ Michela Zilli ${ }^{2}$ \\ Gian Luigi Gigli ${ }^{1,2}$ \\ Mariarosaria Valente ${ }^{1,2}$ \\ 'Department of Neuroscience, S. Maria \\ della Misericordia University Hospital, \\ Udine, Italy; ${ }^{2}$ Department of 'Area \\ MEdica', University of Udine, Udine, Italy
}

Purpose: Although some studies have suggested an association between cardiovascular disease and restless legs syndrome (RLS), the mechanisms underlying this relationship remain unclear. The intima-media thickness (IMT) and vasomotor reactivity are two simple, non-invasive tools to investigate preclinical atherosclerosis and microangiopathy, respectively. The aims of this study were to evaluate carotid IMT and to explore vasomotor reactivity in idiopathic RLS (iRLS) patients.

Patients and Methods: We enrolled 44 iRLS after exclusion of patients with secondary causes of RLS, history of vascular events, known uncontrolled vascular risk factors and other neurological disorders. Forty-four age and sex matched controls were therefore recruited. No significant differences in demographic data and vascular risk factors were found between the two groups. Carotid IMT was measured with a high-resolution B-mode ultrasound on the farwall of common carotid artery, $10 \mathrm{~mm}$ and $30 \mathrm{~mm}$ to the carotid bulb. Vasomotor reactivity to hypo- and hypercapnia was assessed, by right middle cerebral artery transcranial Doppler, accordingly to the changes in peak systolic velocity, peak diastolic velocity and mean blood flow velocity.

Results: Mean IMT was significantly increased in patients with iRLS when measured immediately proximally to carotid bifurcation $(0.73 ; \mathrm{sd}=0.17)$, versus controls $(0.65$; $\mathrm{sd}=0.13) ; \mathrm{p}=0.035$. Patients showed higher cerebrovascular flow velocities (CBFVs) compared to controls. After multivariate analysis, age, hypertension and iRLS proved to be independent IMT predictors.

Conclusion: Increased IMT and higher CBFVs in iRLS support the association of iRLS with vascular damage, possibly through enhanced atherogenesis and sympathetic hyperactivity. However, to clarify a causal relationship, further longitudinal assessment of these parameters is needed, trying to control all their physiological modifying factors.

Keywords: atherosclerosis, carotid disease, stroke, sleep disorders

\section{Introduction}

Restless legs syndrome (RLS) is a common neurological sensory-motor disorder characterized by an uncontrollable urge to move legs, often accompanied by sensory discomfort. Other features include worsening of symptoms at night or with inactivity and development of relief with movement. ${ }^{1}$ RLS can be primary, idiopathic (iRLS), or secondary to anemia, low iron levels, kidney failure, peripheral neuropathy, drugs, etc. ${ }^{2}$ Moreover, few studies showed that stroke as well, notably with a subcortical or brainstem location, is responsible for secondary forms
Correspondence: Francesco Janes Department of Neuroscience, S. Maria della Misericordia University Hospital, Udine, Italy

Tel +390432559020

Fax +390432552719

Email francesco.janes@asufc.sanita.fvg.it 
of RLS. ${ }^{3,4}$ Several data suggest a very complex pathogenetic interplay among multi-transmitter neurochemical pathways in RLS. Their dysfunction seems to be determined both by genetic and acquired factors. ${ }^{5}$ This multifaceted background probably justify the conflicting and often inconsistent evidence on the association between RLS and cardio-vascular disease (CVD). However, this might also be the consequence of the limited number of papers on this topic and the difficulty to exclude frequent comorbid conditions such as hypertension and other vascular risk factors, obstructive sleep apnea, etc. ${ }^{6,7}$ Different possible mechanisms have been postulated linking RLS to the development of CVD. The direct enhancement of atherogenesis is the least likely. The sympathetic over activity, in association with hypertension, seems to be the direct pivotal factor in determining vessels damage. It is still debated which of these two elements actually start the pathological cascade, but their relationship seems bidirectional once established.

IMT provides a surrogate marker of cardiovascular outcomes in clinical trials evaluating the efficacy of cardiovascular risk factors modification. Many studies have successfully applied IMT as a technique to monitor arterial wall alterations based upon its association with cardiovascular risk factors and incident cardiovascular disease. Moreover, increased carotid IMT is a non-invasive marker of systemic atherosclerosis and is related to the risk of future CVD and stroke. ${ }^{8-10}$

Cerebrovascular Vasomotor Reactivity is defined as the percent change in blood flow velocity in response to changes in carbon dioxide, which can act as a powerful vasodilator. $^{11,12}$ Clinically, CVR can be assessed by Transcranial Doppler (TCD) and can be tested by holdbreathing and hyperventilation tests, measuring the ability of the cerebral micro-vessels to dilate and constrict in response to hypercapnia and hypocapnia. Therefore, CVR gives an index of reactivity of the intracranial vessels in response to a stimulus through ventilatory alterations of PaCO2. ${ }^{13}$ Impairment of CVR has been linked to pathologies as obstructive and central sleep apnea, ${ }^{14-16}$ carotid artery stenosis, ${ }^{17}$ hypertension, ${ }^{18}$ congestive heart failure $^{19}$ and cerebral ischemic events. ${ }^{20}$ Moreover, it proved to be an independent predictor of ischemic stroke in some studies. ${ }^{21-23}$ However, all those studies show some conflicting pathophysiological interpretations, probably due to the complexity of physiological responses and to the presence of several modifying factors.
The aim of our study was to investigate the relationship between iRLS and vascular diseases, through the analysis of two parameters: IMT, as a non-invasive marker of systemic atherosclerosis; CVR (through analysis of cerebral blood flow velocity; CBFV), to identify a hypothetical impairment in vascular function and changes in autonomic system. The need to study this relationship rise because even if there is an increasing amount of evidence supporting the association between RLS and vascular diseases, the mechanisms underlying this relationship still remain unclear: whether it enhances atherosclerosis or impairs vascular response, which eventually leads to higher susceptibility to vascular risk factors themselves.

\section{Materials and Methods Participants' Selection}

A screening of patients available on the University of Udine Neurology Unit database, presenting to the "Sleep Disorders" outpatient service or admitted as inpatients, between January 2011 and December 2013, was performed before study enrolment. Participants were then evaluated from June 2014 to April 2016. We included in the study people affected by idiopathic RLS according to international criteria, ${ }^{1}$ aged over 18 years.

Using clinical and laboratory data, we excluded from the patients group, people with iron deficiency, renal failure, pregnancy, history of vascular diseases (stroke, transient ischemic attack, acute myocardial infarction, angina pectoris, history of percutaneous transluminal coronary angioplasty, claudicatio intermittens), untreated and uncontrolled high blood pressure, patients with poor glycemic control, smoking $>20$ cigarettes per day, with carotid plaques, with features of cerebral small vessel disease (as detected by brain structural imaging). Patients with other known neurological disorders, arising from clinical history or suspected after examination, were also excluded.

Clinical history was used to exclude from control group people with anemia, renal failure, pregnancy, vascular diseases, risk factors and other neurological diseases (as already specified above for patients' group).

Group size was determined according to the number of patients evaluated during two years in our sleep-disorders outpatient service. Patients were classified by sex and by age, with assignment to 5-years-age intervals; controls were then selected, without RLS and according to exclusion criteria, among the sanitary personnel of our 
department and their relatives, trying to correspond to patients' distribution.

All participants were evaluated according to the same protocol and provided written informed consent. The study was considered exempt from "Institutional Review Board" (IRB) approval because collected information consisted only in: i. a more detailed medical history than the usual one in an outpatient visit; ii. blood tests that patients with RLS do as standard diagnostic workup; iii. non-invasive echographic imaging. The study was conducted in accordance with the ethical principles for medical research involving human subjects stated in the Declaration of Helsinki.

\section{Variables Collected}

Participants' demographics, information about smoke, alcohol and coffee consumption habits, current treatments (antidepressants, dopaminergic drugs, anti-epileptics, benzodiazepines, etc.) comorbidity, laboratory data and vascular risk factors were collected during a face-to-face interview, conducted by a physician expert in Sleep Medicine. Furthermore, data concerning sleep quality (Epworth Sleep Scale and Pittsburgh Sleep Scale), obstructive sleep apnea syndrome (OSAS) risk (Berlin Questionnaire and polysomnographic study of participants at risk) and RLS features (clinical criteria, RLS Severity Scale, history of familial RLS, onset, distribution and severity of symptoms, etc.) were obtained.

We calculated the body mass index by checking their body weight and their height. Hypertension was defined as a systolic blood pressure $>$ or $=140 \mathrm{mmHg}$ and/or diastolic blood pressure $>$ or $=90 \mathrm{mmHg}$, or as a report of current treatment with anti-hypertensives. Diagnosis of diabetes mellitus was considered according to ADA standard criteria and in case of treatment with anti-diabetic drugs. Hypercholesterolemia was defined as total serum cholesterol $>$ or $=230 \mathrm{mg} / \mathrm{dl}$ or current treatment with cholesterol-lowering drugs. Positivity to "Thyroid disease" refers to both hypothyroidism and hyperthyroidism or normal thyroid function with medications. A plaque was defined as a focal structure that encroached into the arterial lumen at least $0.5 \mathrm{~mm}$ or that was $50 \%$ of the surrounding IMT value, or that had a thickness $>1.5 \mathrm{~mm}$ as measured from the media-adventitia interface to the intima-lumen interface.

\section{Carotid IMT Measurements}

Measurement of carotid intima-media thickness (IMT) was performed by high-resolution B-mode ultrasonography

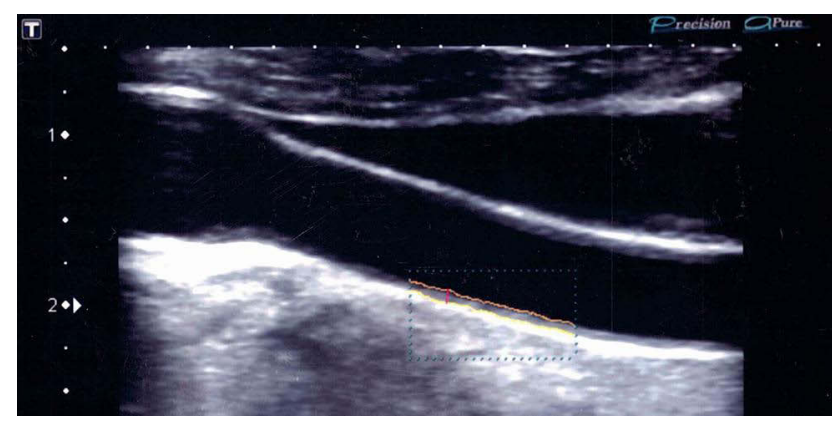

Figure I Intima media thickness.

Notes: Here is reported a picture of an IMT measurement. In this example, the IMT has been measured at $\mathrm{I} \mathrm{cm}$ from carotid bifurcation, along the carotid farwalland within a $\mathrm{Icm}$ width Rol.

Abbreviations: IMT, Intima Media Thickness; Rol, Region of Interest.

(Toshiba Aplio 400), using a $7 \mathrm{MHz}$ linear ultrasound transducer, according to the guidelines of the Mannheim IMT Consensus. ${ }^{24}$ The IMT was defined as the distance from the leading edge of the lumen-intima interface to the leading edge of the media-adventitia interface of the far wall. $^{25}$ A longitudinal image of the common carotid artery (CCA), strictly perpendicular to the ultrasound beam, was obtained proximally to the carotid bulb, with the patient in a supine position. After clearly identifying the double-line pattern, we proceed to measure the CCA-IMT on the far wall of the left and of the right side, using a rangecalibrated trackball, approximately $10 \mathrm{~mm}$ and $30 \mathrm{~mm}$ to the carotid bulb. An automated system (USAI-790A/EL Auto IMT - Toshiba Medical System(C) provided the measurement of mean IMT on both sides. Moreover, automated system could provide the mean maximum value of 100 measurements performed on a 5-mm segment of CCA instantaneously. Figure 1 shows an example of measurement methodology. All these and the following sonographic measurements were carried out by an experienced neurosonologist, blinded to the subjects' group assignment.

\section{Transcranial Doppler Assessment}

Vasomotor reactivity was measured as follows. Participants laid in supine position and were asked to breathe normally for 1 minute; the proximal part of the right middle cerebral artery (MCA) was then insonated through trans-temporal approach with a $2 \mathrm{MHz}$ probe at a 5 to $6 \mathrm{~cm}$ depth and the Doppler signal was optimized by adjusting probe position precisely. Examinations were performed by a single experienced neurosonologist. Peak systolic velocity, end diastolic velocity and mean blood flow velocity were evaluated at rest (as baseline), after 30-seconds or maximum-tolerated 
breath-holding (after a normal inspiration to avoid a Valsalva manoeuvre) and during hyperventilation (when stable flow was obtained). Flow Velocity Excursion (F.V.E.) was defined as the difference between baseline values and post-manoeuvre values. We chose to record right MCA only according to published evidence of substantial equivalence between the two sides. ${ }^{26}$ People with absent or suboptimal acoustic window were not included in the analysis.

\section{Statistical Analysis}

A power analysis was not performed. Demographic and clinical data are presented as mean \pm sd or as absolute number (and percentage out of total) according to the nature of the variable itself. In the univariate analysis a Student's $t$-test for independent samples was used for normally distributed continuous variables; a chi-square test was used for categorical variables. A p-value $<0.05$ was considered as limit of significance for both tests. A multiple-regression model was used to explore the interplay among different variables in predicting IMT value. Analysis was performed using SPSS 13.0.

\section{Results}

From June 2014 to April 2016, we enrolled 48 consecutive patients with iRLS; 4 patients were excluded after enrolment because of emerging exclusion criteria ( 1 for evidence of carotid plaque; 1 for a history of a possible TIA; 2 due to missing of a reliable medical history) and 44 patients were finally included in the analysis and compared to 44 controls. Clinical and anthropometric characteristics of all the participants are shown in Table 1. No significant differences in demographic and vascular risk factors data were found between patients with iRLS and controls. The mean age was $57.6 \pm 12.6$ years in the iRLS group and $56.5 \pm 11.7$ years in the control group. Thirty women were enrolled in the iRLS group (54.5\%) and 25 women were selected in the control group (45.5\%). A mean BMI of $26.0 \pm 4.1$ was measured in iRLS group and a mean BMI of $24.7 \pm 2.8$ in the control group; the difference in BMI, though not significant ( $\mathrm{p}=0.09$ ), is mainly due to 6 patients with a BMI $>30$ in the iRLS group; on the contrary, no one in the control group had a BMI $>$ or $=30$.

Table 2 shows IMT values: we found significant difference between the two group in mean and maximum IMT when measured proximally (at $1 \mathrm{~cm}$ ) to carotid bifurcation, while no significant difference was observed in mean and maximum IMT values at $3 \mathrm{~cm}$ far from carotid bifurcation. We found a strong correlation between age and mean IMT values $(\mathrm{r}=0.65 ; \mathrm{p}<0.001$ among all participants and $r=0.77$; $p<0.001$ considering patients with iRLS only). A significant correlation is present as well between RLS duration (years from onset) and mean IMT values $(r=0.35 ; p=0.02)$. If a "lifetime exposure" to RLS was calculated (as a ratio between years from RLS onset and age) this variable was not significantly associated with mean IMT values $(\mathrm{r}=0.18 ; \mathrm{p}=0.28)$. These results are shown in Figure 2. Similar results are also provided in the same figure for maximum IMT. RLS severity was not correlated with mean IMT $(r=0.56 ; \mathrm{p}=0.72)$.

Table I Baseline Characteristics

\begin{tabular}{|l|c|c|c|}
\hline & $\begin{array}{c}\text { i-RLS } \\
(\mathbf{n = 4 4 )}\end{array}$ & $\begin{array}{c}\text { Controls } \\
\text { (n=44) }\end{array}$ & $\begin{array}{c}\text { p-value } \\
\text { t-test (95\% C.I.) or } \boldsymbol{X}^{\mathbf{2}}\end{array}$ \\
\hline Age, mean (sd) & $57.6(12.6)$ & $56.5(11.7)$ & 0.69 \\
Sex, n-female (\%) & $30 / 44(54.5)$ & $25 / 44(45.5)$ & 0.38 \\
BMI, mean (sd) & $26.0(4.1)$ & $24.7(2.8)$ & 0.09 \\
Smoke $\mathrm{n}(\%)$ & $12 / 44(27.3)$ & $15 / 44(34.1)$ & 0.64 \\
Alcohol n(\%) & $14 / 44(31.8)$ & $16 / 43(37.2)$ & 0.66 \\
Coffee $\mathrm{n}(\%)$ & $38 / 44(86.4)$ & $34 / 44(77.3)$ & 0.47 \\
Thyroid disease & $8 / 44(18.2)$ & $6 / 44(13.6)$ & 0.77 \\
Hypercholesterolemia & $7 / 44(15.9)$ & $8 / 43(18.6)$ & 0.78 \\
Hypertension & $13 / 44(29.5)$ & $7 / 43(16.3)$ & 0.20 \\
OSAS & $10 / 44(22.7)$ & $8 / 44(18.2)$ & - \\
RLS - severity scale & $21.8(6.0)$ & - & - \\
RLS - years from onset median (min-max) & $5(1-45)$ & - & - \\
Dopaminergic treatment n(\%) & $30 / 44(68.2)$ & $0 / 44$ & - \\
\hline
\end{tabular}

Note: *The \% here reported refers to the proportion of patients treated at the time of study evaluation. Abbreviations: BMI, Body Mass Index; OSAS, Obstructive Sleep Apnea Syndrome. 
Table 2 Carotid Intima Media Thickness

\begin{tabular}{|l|c|c|c|}
\hline & $\begin{array}{c}\text { i-RLS } \\
(\mathbf{n = 4 4 )}\end{array}$ & $\begin{array}{c}\text { Controls } \\
\mathbf{( n = 4 4 )}\end{array}$ & $\begin{array}{c}\text { p-value } \\
\text { t-test - 95\% C.I. }\end{array}$ \\
\hline Mean IMT - Icm & $0.73(0.17)$ & $0.65(0.13)$ & $0.035^{*}$ \\
Max IMT - I cm & $0.88(0.20)$ & $0.78(0.15)$ & $0.016^{*}$ \\
Mean IMT - 3 cm & $0.71(0.14)$ & $0.66(0.10)$ & 0.117 \\
Max IMT - 3 cm & $0.84(0.17)$ & $0.79(0.13)$ & 0.120 \\
\hline
\end{tabular}

Notes: IMT at $\mathrm{Icm}$ and $3 \mathrm{~cm}$ from Carotid Bifurcation respectively; *Marks significant $\mathrm{p}$ values $<0.05$.

Abbreviation: IMT, Intima Media Thickness.

We tested in a multiple-regression model the role of Age, BMI, Hypertension, OSAS and diagnosis of iRLS as predictors of mean-IMT increase (Table 3): in this model Age is the strongest predictor, RLS is still significantly associated to IMT levels and hypertension is marginally significant.

Nine subjects with a poor acoustic window, 4 in the iRLS group and 5 in the control group, were excluded from TCD assessment. Table 4 shows mean and maximum middle cerebral artery (MCA) velocities at basal conditions, after breath-holding test and during hyperpnoea in the two groups. A statistically significant difference between the two groups was found in all the three settings. Overall blood Flow Velocity Excursion (F.V.E.) is significantly increased in patients versus controls (mean F.V.E. = $30.3 \pm 12.8 \mathrm{~cm} / \mathrm{s}$ versus $15.0 \pm 12.5 \mathrm{~cm} / \mathrm{s}$ ), mainly due to a different pattern of flow velocity change in hold-breath test between patients (mean F.V.E. $=12.1 \pm 10.2$ ) and controls (mean F.V.E. $=-2.3 \pm 12.4$ ).

\section{Discussion}

In this study, we found that IMT, measured proximally to carotid bifurcation $(1 \mathrm{~cm})$, is increased in iRLS patients, compared to age- and sex-matched controls; we also found that age, RLS and hypertension were independent IMT predictors after multivariate analysis. According to our data, no statistically significant differences in IMT at $3 \mathrm{~cm}$ from carotid bifurcation have been observed. Compared to IMT values at $1 \mathrm{~cm}$ from carotid bifurcation, measurement at $3 \mathrm{~cm}$ could be less exposed to low shearstress and blood pressure damage, as suggested by few studies. ${ }^{27}$ Our results appear to be consistent with other studies on RLS supporting a strict association between CVD and iRLS, in particular when associated with isolated periodic limb movements (PLMs), brief muscle activations occurring at regular intervals. ${ }^{6}$ Nevertheless, our results do not confirm data published in 2012 by Park, ${ }^{28}$ which showed a maximum value of IMT in patients with iRLS lower than in controls, after adjustment for vascular risk factors. In Park's paper, patients with RLS and PLMs were excluded. On our hand, we did not systematically investigate the presence of PLMs with laboratory polysomnography (PSG) in all participants. However: i) PMLs are extremely common in patients with RLS, up to $90 \%$ of cases; ${ }^{29}$ ii) They are not specific for a diagnosis of RLS (being associated with post-traumatic stress disorder, narcolepsy, renal disease, congestive heart failure, alcoholism, mood disorders, Parkinson's disease, idiopathic REM sleep behavior disorder, diabetes mellitus, normal aging, etc.) iii) To exclude PLMs in iRLS is difficult with a single recording. Given these considerations, we think it would be much more informative to correlate PLMs frequency in PSG to IMT or other variables, rather than including only iRLS presumed to be negative for PLMs.

It is likely that the slightly more elevated BMI in the iRLS group (6 patients with BMI $>$ or $=30$ ), not statistically significant, should not have contributed importantly to determine a significant difference between cases and controls IMTs. In fact, although BMI positively correlates with IMT $(r=0.28 ; p=0.01)$, it was not an independent predictor of IMT value in the multiple regression model. Compared with Park study, we have tried to better characterize RLS in patients, recording severity and disease burden (in years from onset to evaluation). A significant correlation is present between RLS duration (years from onset) and IMT values, but If a "lifetime exposure" to RLS was calculated (as shown in Figure 2) this variable is not significantly associated with IMT values. This result seems to be affected by the strong correlation between age and IMT (Table 2). RLS severity was not significantly associated with mean IMT. The majority of patients ranged between moderate and severe RLS, indicating at least suboptimal treatment. However, the score here reported refers to the time of evaluation only, thus not reflecting the past years trend: this might justify the apparent inconsistency with the other results and actually hamper speculations on the relationship between RLS severity and IMT. Moreover, a detailed and reliable medication history was lacking in the majority of our patients. This point precludes clear hypothesis about the consequences of RLS treatment (above all if effective or not) on IMT progression in our sample.

Altogether, the results above reported do not clarify a causal relationship between independent risk factors, but they hint at the role of hypertension and iRLS as speed-up 
A

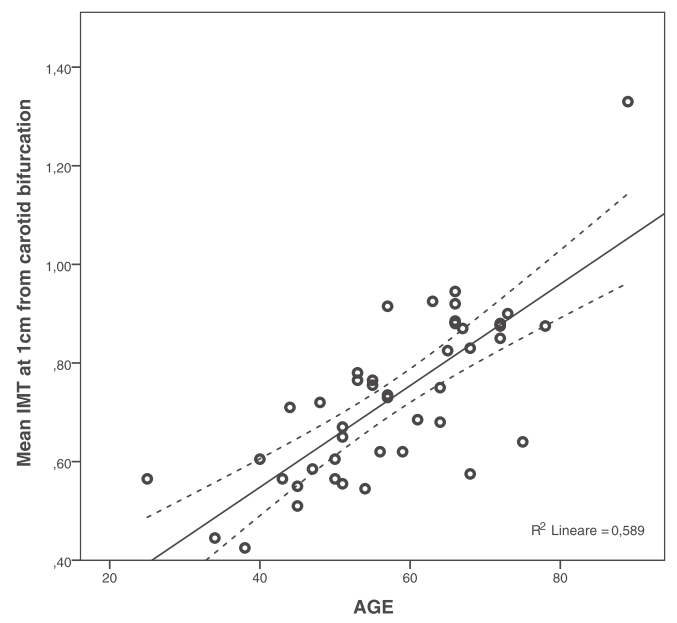

B

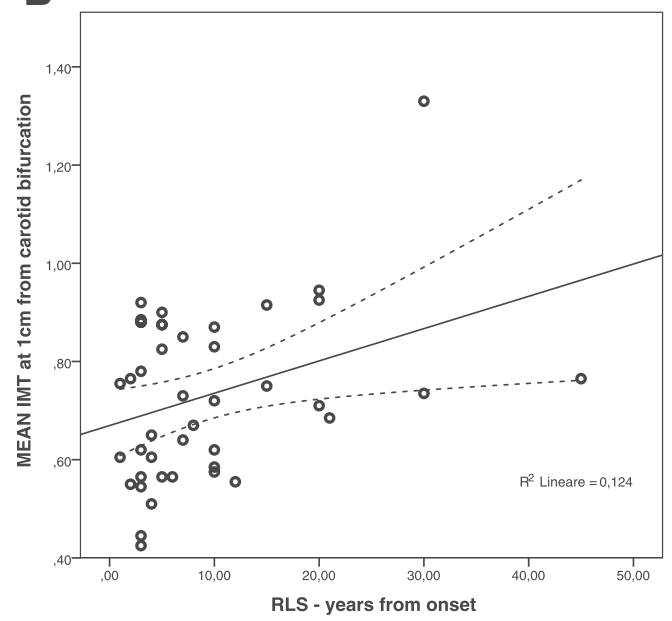

C

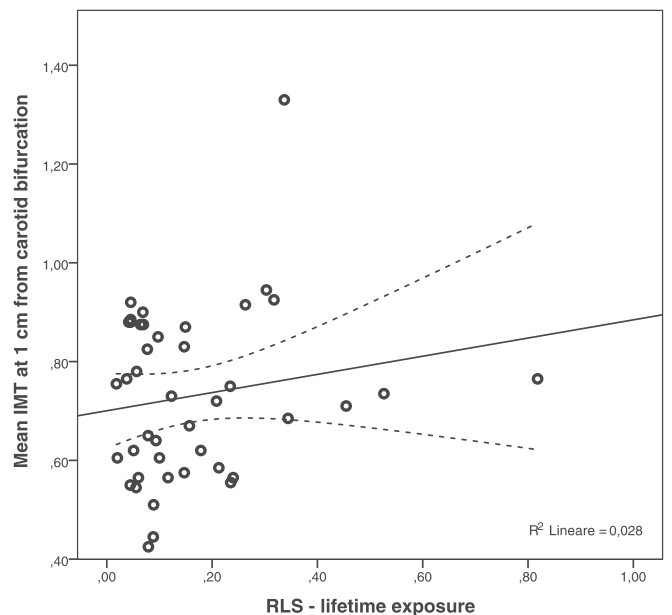

D

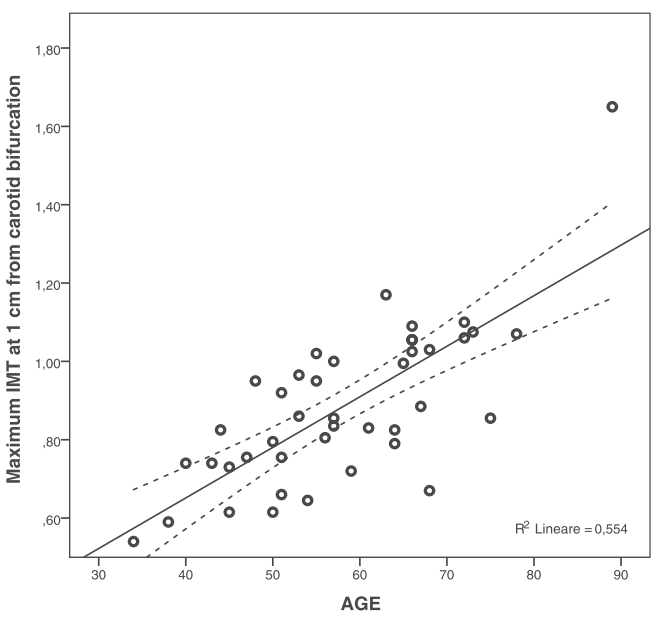

E

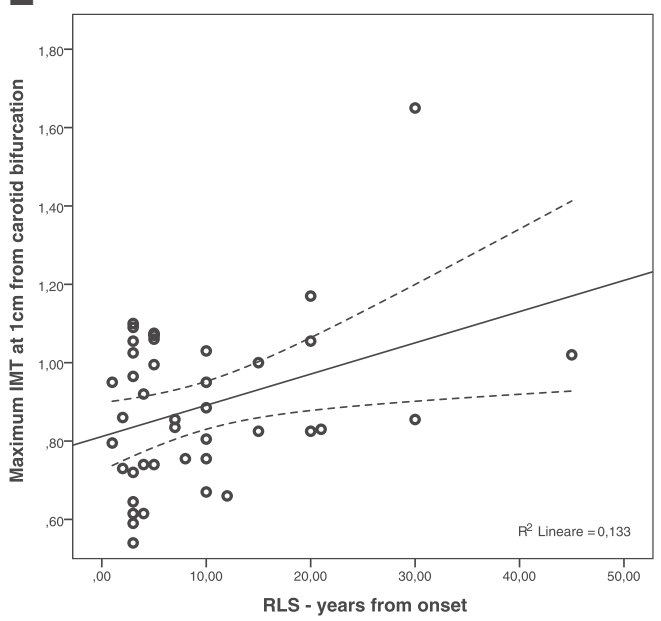

$\mathbf{F}$

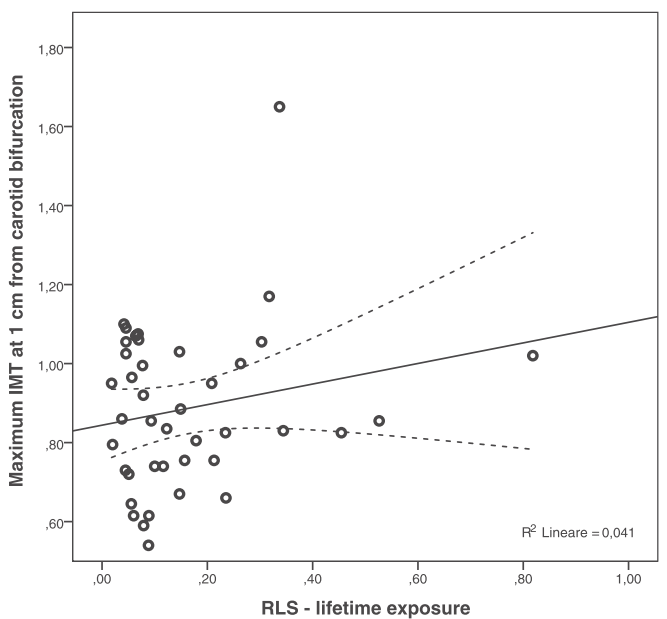

Figure 2 Intima media thickness and clinical features.

Notes: Relationship between mean IMT (at Icm from carotid bifurcation) and age (A), RLS duration (B) and RLS lifetime exposure (C); between max IMT (at Icm from carotid bifurcation) and age (D), RLS duration (E) and RLS lifetime exposure (F) Abbreviations: IMT, Intima Media Thickness; RLS, Restless Legs Syndrome. 
Table 3 Multiple Regression on Predicting Variables of Carotid Mean IMT

\begin{tabular}{|l|l|l|l|l|}
\hline & B & Std. Error & Beta & Sig. \\
\hline RLS & 0.062 & 0.028 & 0.193 & 0.030 \\
Age & 0.007 & 0.001 & 0.545 & $<0.001$ \\
BMI & 0.001 & 0.004 & 0.026 & 0.781 \\
OSAS & -0.005 & 0.034 & -0.14 & 0.876 \\
Hypertension & 0.074 & 0.036 & 0.204 & 0.044 \\
Constant & 0.194 & 0.110 & & 0.086 \\
\hline
\end{tabular}

Abbreviations: BMI, Body Mass Index; OSAS, Obstructive Sleep Apnea Syndrome.

factors on IMT increase across aging. Future studies should take into consideration prospectively, lifetime exposure to RLS, its severity and treatment.

The second aim of the study was to investigate CVR in patients with iRLS, supposing that cerebral microvascular impairment of tone adaptation in RLS could work together with other vascular risk factors, rather than directly enhancing atherosclerotic progression. Under basal conditions, cerebral vessels rapidly adapt to changes in perfusion pressure (cerebral autoregulation), regional metabolic requirements of the brain (neurovascular coupling), autonomic neural activity, ${ }^{30,31}$ and humoral factors (cerebrovascular reactivity), in particular the partial pressure of arterial carbon dioxide (PaCO2). Regulation of $\mathrm{CBF}$ is highly controlled and involves a wide spectrum of regulatory mechanisms that together work to provide adequate oxygen and nutrient supply: ${ }^{32-38}$ This complex system seems to explain the contradictory results of some studies. Our research focused on CVR and shows significantly higher MCA velocities in iRLS compared to controls under basal condition, after hold-breath test and after hyperpnoea. This seems to be consistent with the known hypothesis that the loss of inhibitory function by hypothalamic dopaminergic neurons of the A11 area is able to shift the balance of descending control pathways toward excitation at the sympathetic preganglionic fibers level, although some recent data suggest that the sympathetic system exerts its influence on CO2-related vasoreactivity only during hypocapnia. ${ }^{39}$ Interestingly, the concomitant increase of CBFVs and IMT in our patients seems consistent with the results of other studies in which TCD hemodynamic changes were found in preclinical stages of cerebrovascular disease. ${ }^{40,41}$ This strengthens the hypotheses that iRLS could represent a facilitator of cerebrovascular damage and that it could increase the risk of both cerebrovascular events and vascular cognitive impairment. However, these results should be considered cautiously since we did not control for several variability factors such as concurrent medications used by patients and above all circadian fluctuation in CBFV. In fact, blood flow velocities are lower in the morning and higher in the afternoon and in the evening. ${ }^{37}$ We did not record TCD time nor plan a specific TCD time schedule. Moreover, hypercapnia was evaluated through maximal tolerated apnea, regardless of its complex-shaped response

Table 4 MCA Flow Velocities by TCD

\begin{tabular}{|c|c|c|c|c|}
\hline \multicolumn{2}{|l|}{ F.V. $(\mathrm{cm} / \mathrm{s})$} & \multirow{3}{*}{$\begin{array}{c}\begin{array}{c}\mathrm{i}-\mathrm{RLS} \\
(\mathrm{n}=40)\end{array} \\
53.6(11.7) \\
79.4(17.5)\end{array}$} & \multirow{3}{*}{$\begin{array}{c}\begin{array}{c}\text { Controls } \\
(n=39)\end{array} \\
43.9(13.6) \\
68.6(20.1)\end{array}$} & \multirow{3}{*}{$\begin{array}{c}\begin{array}{c}\text { p-value } \\
\text { t-test - } 95 \% \text { C.I. }\end{array} \\
0.001^{*} \\
0.012^{*}\end{array}$} \\
\hline Basal & Mean F.V. & & & \\
\hline & Max F.V. & & & \\
\hline \multirow[t]{2}{*}{ Max apnea/Hold- breathing } & Mean F.V. & 65.7 (I7.5) & $41.6(18.1)$ & $<0.001 *$ \\
\hline & Max F.V. & $91.9(24.5)$ & $63.6(25.2)$ & $<0.001 *$ \\
\hline \multirow[t]{2}{*}{ Hyperpnoea } & Mean F.V. & $35.4(9.0)$ & $26.6(9.8)$ & $<0.00 I^{*}$ \\
\hline & Max F.V. & $62.2(15.9)$ & $48.2(19.6)$ & $0.001 *$ \\
\hline \multirow[t]{2}{*}{ Overall Excursion } & Mean F.V.E. & $30.3(12.8)$ & $15.0(11.8)$ & $<0.001 *$ \\
\hline & Max F.V.E. & $29.7(15.8)$ & I5.3 (I2.5) & $<0.001 *$ \\
\hline Max apnea/ & Mean F.V.E. & $12.1(10.2)$ & $-2.3(12.4)$ & $<0.001 *$ \\
\hline Hold- breathing & Max F.V.E. & $12.5(13.6)$ & $-5.0(14.3)$ & $<0.001 *$ \\
\hline \multirow[t]{2}{*}{ Hyperpnoea } & Mean F.V.E. & $18.1(8.1)$ & $17.4(8.5)$ & 0.67 \\
\hline & Max F.V.E. & I7.2(|I.0) & $20.4(8.8)$ & 0.16 \\
\hline
\end{tabular}

Note: *Marks significant $p$ values $<0.05$.

Abbreviations: MCA, Middle Cerebral Artery; F.V., Flow Velocity; E., Excursion. 
which can be divided into four phases: ${ }^{38}$ differently from patients, in the control group mean and maximum MCA velocities decrease after breath-holding test compared to basal conditions. This might be explained by a different pattern of response to hypercapnia in the two groups, but it could also be the effect of differences in test setting (below, under limitations). Consequently, we can speculate that sympathetic hyperactivity could have determined higher FVs in iRLS than in controls, but we could not demonstrate vasoreactivity impairment in the former group.

Finally, our study has several limitations: a) The number of study participants was relatively small; b) No systematic polysomnographic data were collected in all participants to detect presence and number of PLMs in the two groups investigated; c) Electromyographic study was not systematically performed in RLS group to assess possible polyneuropathies; d) Breath-holding tests assessed by TCD should have taken into account time-dependent CBFV changes during measurement so that a "Breath-holding index" could have been calculated; e) Measurement of CBFV should be obtained at the same time during the day to cut out circadian rhythm variability; f) Pulsatility index and Resistivity index were not recorded; g) systolic and diastolic pressure could have been measured at the same time TCD was performed; h) vertebra-basilar system and bilateral MCA velocities were not explored; i) we also cannot exclude selection biases in subjects' enrollment, in relation to the specific inclusion and exclusion criteria we chose; j) we cannot exclude the effect that drugs taken by patients - data not-recorded in this study - would have had on the neurosonologic measurements.

Due to these limitations our findings should be considered cautiously. Further studies are necessary in patients with iRLS to confirm them, particularly about CVR evaluation.

\section{Conclusion}

We showed that iRLS is associated with an increased IMT and higher CBFVs, compared to controls. The crosssectional nature of this study precludes to demonstrate causal relationships among associated factors, but these results support the role of iRLS as risk cofactor for vascular damage. Further longitudinal studies on CVR and preclinical features of vascular damage are needed to explore mechanisms of vascular disease in patients with iRLS. These studies should try to control for all its several physiological and pathophysiological modifying factors.

\section{Acknowledgments}

The authors are thankful to Prof. Marina Diomedi and Prof. Mauro Silvestrini for kindly reviewing the manuscript.

\section{Disclosure}

The authors report no conflicts of interest in this work.

\section{References}

1. Allen RP, Picchietti DL, Garcia-Borreguero D, et al. Restless legs syndrome/Willis-Ekbom disease diagnostic criteria: updated International Restless Legs Syndrome Study Group (IRLSSG) consensus criteria - history, rationale, description, and significance. Sleep Med. 2014;15:860-873. doi:10.1016/j.sleep.2014.03.02

2. Valente M, Janes F, Russo V, et al. Prevalence of restless legs syndrome in migraine patients: a case-control study. analysis of risk factors for restless legs syndrome in migraine patients. Headache. 2017;57(7):1088-1095. doi:10.1111/head.13124

3. Wu X, Xu J, Lu B. Acute post-stroke restless legs syndrome: the body of caudate nucleus considerations. Sleep Med. 2020;70:66-70. doi:10.1016/j.sleep.2019.11.1253

4. Lee SJ, Kim JS, Song IU, An JY, Kim YI, Lee KS. Poststroke restless legs syndrome and lesion location: anatomical considerations. Mov Disorders. 2009;24:77-84. doi:10.1002/mds.22303

5. Lanza G, Ferri R. The neurophysiology of hyperarousal in RLS: hints for a role of glutamate/GABA. Adv Pharmacol. 2019;84:101-119. doi:10.1016/bs.apha.2018.12.002

6. Ferini-Strambi L, Walters AS, Sica D. The relationship among restless legs syndrome (Willis -Ekborm Disease), hypertension, ossiblecular disease and cerebrovascular disease. $J$ Neurol. 2014;261:1051-1068. doi:10.1007/s00415-013-7065-1

7. Gottlieb DJ, Somers VK, Punjabi NM, Winkelman JW. Restless legs syndrome and cardiovascular disease: a research roadmap. Sleep Med. 2017;31:10-17. doi:10.1016/j.sleep.2016.08.008

8. Lorenz MW, von Kegler S, Steinmetz H, Markus HS, Sitzer M. Carotid intima-media thickening indicates a higher vascular risk across a wide age range: prospective data from the Carotid Atherosclerosis Progression Study (CAPS). Stroke. 2006;37:87-92. doi:10.1161/01.STR.0000196964.24024.ea

9. Van der Meer IM, Iglesias Del Sol A, Hak AE, Bots ML, Hofman A, Witteman JC. Risk factors for progression of atherosclerosis measured at multiple sites in the arterial tree: the Rotterdam Study. Stroke. 2003;34(10):2374-2379. doi:10.1161/01.STR.0000088 643.07108 .19

10. Lorenz MW, Markus S, Bots ML, Rosvall M, Sitzer M. A systematic review and meta-analysis: prediction of clinical cardiovascular events with carotid intima-media thickness. Circulation. 2007;115:459-467. doi:10.1161/CIRCULATIONAHA.106.628875

11. Sloan MA, Alexandrov AV, Tegeler $\mathrm{CH}$, et al. Technology assessment subcommittee of the American Academy of neurology. assessment: transcranial Doppler ultrasonography. Report of the therapeutics and technology assessment subcommittee of the American Academy of neurology. Neurology. 2004;62:1468-1481. doi:10.1212/wnl.62.9.1468

12. Markus HS, Harrison MJ. Estimation of cerebrovascular reactivity using transcranial Doppler, including the use of breath-holding as the vasodilatory stimulus. Stroke. 1992;23:668-673. doi:10.1161/01. str.23.5.668

13. Willie CK, Colino FL, Bailey DM, et al. Utility of transcranial Doppler ultrasound for the integrative assessment of cerebrovascular function. J Neurosci Methods. 2011;196:221-237. doi:10.1016/j. jneumeth.2011.01.011 
14. Reichmuth K, Dopp JM, Barczi SR, et al. Impaired vascular regulation in patients with obstructive sleep apnea: effects of cpap treatment. Am J Respir Crit Care Med. 2009;180(11):1143-1150. doi:10.1164/rccm.200903-0393OC

15. Burgess KR, Fan JL, Peebles KC, et al. Exacerbation of obstructive sleep apnea by oral indomethacin. Chest. 2010;137:707-710. doi:10.1378/chest.09-1329

16. Hill CM, Hogan A, Kirkham FJ, et al. Increased cerebral blood flow velocity in children with mild sleep-disorder breathing: a possible association with abnormal neuropsychological function. Pediatrics. 2006;118(4):e1100-e1108. doi:10.1542/peds.2006-0092

17. Widder B, Kleiser B, Krapf H. Course of cerebrovascular reactivity in patients with carotid artery occlusions. Stroke. 1994;25:1963-1967. doi:10.1161/01.str.25.10.1963

18. Serrador JM, Sorond FA, Vyas M, Gagnon M, Iloputaife ID, Lipsitz LA. Cerebral pressure-flow relations in hypertensive elderly humans: transfer gain in different frequency domains. $J$ Appl Physiol. 2005;98:151-159. doi:10.1152/japplphysiol.00471.2004

19. Xie A, Skatrud JB, Khayat R, Dempsey JA, Morgan B, Russell D. Cerebrovascular response to carbon dioxide in patients with congestive heart failure. AmJ Respir Crit Care Med. 2005;172:371-378. doi:10.1164/rccm.200406-807OC

20. Wijnhoud AD, Koudstaal PJ, Dippel DW. Relationships of transcranial blood flow Doppler parameters with major vascular risk factors: tcd study in patients with a recent tia or nondisabling ischemic stroke. J Clin Ultrasound. 2006;34:70-76. doi:10.1002/jcu.20193

21. Silvestrini M, Vernieri F, Pasqualetti P, et al. Impaired cerebral vasoreactivity and risk of stroke in patients with asymptomatic carotid artery stenosis. JAMA. 2000;283:2122-2127. doi:10.1001/jama.283.16.2122

22. Markus H, Cullinane M. Severely impaired cerebrovascular reactivity predicts stroke and tia risk in patients with carotid artery stenosis and occlusion. Brain. 2001;124:457-467. doi:10.1093/brain/124.3.457

23. Vernieri F, Pasqualetti P, Matteis M, et al. Effect of collateral blood flow and cerebral vasomotor reactivity on the outcome of carotid artery occlusion. Stroke. 2001;32:1552-1558. doi:10.1161/01.str.32.7.1552

24. Touboul PJ, Hennerici MG, Meairs S, et al. Mannheim carotid intima-media thickness consensus (2004-2006). Cerebrovasc Dis. 2007;23:75-80. doi:10.1159/000097034

25. Meijer R, Grobee DE, Bots ML. Mannheim consensus on carotid intima-media thickness: opposite and complementary points of view. Cerebrovasc Dis. 2006;21:415-416. doi:10.1159/000092129

26. Rosengarten B, Deppe M, Kaps M, Klingelhöfer J. Methodological aspects of functional transcranial Doppler sonography and recommendations for simultaneous EEG recording. Ultrasound Med Biol. 2012;38(6):989-996. doi:10.1016/j.ultrasmedbio.2012.02.027

27. Augst AD, Ariff BM, Thom SM, Xu XY, Hughes AD. Analysis of complex flow and the relationship between blood pressure, wall shear stress, and intima-media thickness in the human carotid artery. $\mathrm{Am}$ J Physiol Heart Circ Physiol. 2007;293(2):H1031-7. doi:10.1152/ ajpheart.00989.2006
28. Park HP, Han SW, Biak JS. Carotid intima-media thickness in patients with idiopathic restless syndrome. Eur Neurol. 2012;67:321-325. doi:10.1159/000334869

29. Rye DB, Trotti LM. Restless legs syndrome and periodic leg movements of sleep. Neurologic Clin. 2012;30:1137-1166. doi:10.1016/j. ncl.2012.08.004

30. Cassaglia P, Griffiths R, Walker A. Cerebral sympathetic nerve activity has a major regulatory role in the cerebral circulation in rem sleep. J Appl Physiol. 2009;106:1050-1056. doi:10.1152/ japplphysiol.91349.2008

31. Cassaglia PA, Griffiths RI, Walker AM. Sympathetic withdrawal augments cerebral blood flow during acute hypercapnia in sleeping lambs. Sleep. 2008;31:1729-1734. doi:10.1093/sleep/31.12.1729

32. Ainslie P, Duffin J. Integration of cerebrovascular $\mathrm{CO} 2$ reactivity and chemoreflex control of breathing: mechanisms of regulation, measurement, and interpretation. AJP: Regulat Integrat Comp Physiol. 2009;296:R1473-95. doi:10.1152/ajpregu.91008.2008

33. Ainslie P, Tzeng Y. On the regulation of the blood supply to the brain: old age concepts and new age ideas. J Appl Physiol. 2010;108:1447-1449. doi:10.1152/japplphysiol.00257.2010

34. Edvinsson L, Krause DN. Cerebral Blood Flow and Metabolism. Philadelphia: Lippincott, Williams \& Wilkins; 2002.

35. Lucas SJ, Tzeng YC, Galvin SD, Thomas KN, Ogoh S, Ainslie PN. Influence of changes in blood pressure on cerebral perfusion and oxygenation. Hypertension. 2010;55:698-705. doi:10.1161/ HYPERTENSIONAHA.109.146290

36. Ogoh S, Ainslie PN. Regulatory mechanisms of cerebral blood flow during exercise: new concepts. Exercise Sport Sci Rev. 2009;37:123-129. doi:10.1097/JES.0b013e3181aa64d7

37. Panerai RB, Dawson SL, Potter JF. Linear and nonlinear analysis of human dynamic cerebral autoregulation. Am J Physiol. 1999;277: H1089-99. doi:10.1152/ajpheart.1999.277.3.H1089

38. Querido JS, Sheel AW. Regulation of cerebral blood flow during exercise. Sports Med. 2007;37:765-782. doi:10.2165/00007256200737090-00002

39. Peebles KC, Ball OG, MacRae BA, Horsman HM, Tzeng YC. Sympathetic regulation of the human cerebrovascular response to carbon dioxide. J Appl Physiol. 2012;113:700-706. doi:10.1152/ japplphysiol.00614.2012

40. Vinciguerra L, Lanza G, Puglisi V, et al. Transcranial Doppler ultrasound in vascular cognitive impairment-no dementia. PLoS One. 2019;14(4):e0216162. doi:10.1371/journal.pone.0216162

41. Vagli C, Fisicaro F, Vinciguerra L, et al. Cerebral hemodynamic changes to transcranial Doppler in asymptomatic patients with Fabry's disease. Brain Sci. 2020;10(8):546. doi:10.3390/ brainsci 10080546

\section{Publish your work in this journal}

Nature and Science of Sleep is an international, peer-reviewed, open access journal covering all aspects of sleep science and sleep medicine, including the neurophysiology and functions of sleep, the genetics of sleep, sleep and society, biological rhythms, dreaming, sleep disorders and therapy, and strategies to optimize healthy sleep.
The manuscript management system is completely online and includes a very quick and fair peer-review system, which is all easy to use. Visit http://www.dovepress.com/testimonials.php to read real quotes from published authors. 